(C) The Authors 2019. This is an Open Access article, distributed under the terms of the Creative Commons Attribution licence (http:// creativecommons.org/licenses/by/4.0/), which permits unrestricted re-use, distribution, and reproduction in any medium, provided the original work is properly cited.

\title{
Early nutrition in combination with polymorphisms in fatty acid desaturase gene cluster modulate fatty acid composition of cheek cells' glycerophospholipids in school-age children
}

\author{
Cristina Martínez-Zaldívar ${ }^{1}$, Hatim Azaryah ${ }^{1}$, José A. García-Santos ${ }^{1}$, Hans Demmelmair ${ }^{2}$, Signe Altmäe $e^{1,3}$, \\ Eva Reischl ${ }^{4}$, Peter Rzehak ${ }^{2}$, Berthold Koletzko ${ }^{2}$, Cristina Campoy ${ }^{1,5,6 *}$ and the NUHEAL team \\ ${ }^{1}$ EURISTIKOS Excellence Centre for Paediatric Research, University of Granada, Avda de la Investigación 11, 18016, \\ Granada, Spain \\ ${ }^{2}$ Division of Metabolic and Nutritional Medicine, Dr. von Hauner Children's Hospital, Ludwig-Maximilians-University of \\ Munich Medical Centre, Lindwurmstr, 4, D-80337 Munich, Germany \\ ${ }^{3}$ Department of Biochemistry and Molecular Biology, Faculty of Sciences, University of Granada, 18016, Granada, Spain \\ ${ }^{4}$ Research Unit of Molecular Epidemiology, Institute of Epidemiology II, Helmholtz Zentrum Muenchen, D-85764 Neuherberg, \\ Munich, Germany \\ ${ }^{5}$ Department of Paediatrics, University of Granada, Avda de la Investigación 11, 18016, Granada, Spain \\ ${ }^{6}$ Network of Biomedical Research Centres on Epidemiology and Public Health (CIBERESP), Institute Carlos III, Madrid, Spain
}

(Submitted 7 February 2018 - Final revision received 5 August 2018 - Accepted 7 August 2018)

\section{Abstract}

Variants in the human genes of fatty acid (FA) desaturase 1 (FADS1), 2 (FADS2) and 3 (FADS3) are associated with PUFA blood levels. We explored if maternal prenatal supplementation and children's genetic variation in seventeen SNP of the FADS1, FADS2 and FADS3 gene cluster influence twenty-one of the most relevant cheek cells' derived FA in glycerophospholipids (GPL-FA). The study was conducted in 147 Spanish and German mother-children pairs participating in the Nutraceuticals for a Healthier Life (NUHEAL) study at 8, 9 and 9.5 years. Linear and mixed model longitudinal regression analyses were performed. Maternal fish-oil (FO) or FO+5-methyltetrahydrofolate (5-MTHF) supplementation during pregnancy was associated with a significant decrease of arachidonic acid (AA) concentrations in cheek cell GPL in the offspring, from 8 to 9.5 years; furthermore, maternal FO + 5-MTHF supplementation was associated with higher $n$ - 6 docosapentaenoic acid concentrations in their children at age 8 years. FADS1 rs174556 polymorphism and different FADS2 genotypes were associated with higher concentrations of linoleic and $\alpha$-linolenic acids in children; moreover, some FADS 2 genotypes determined lower AA concentrations in children's cheek cells. It is suggested an interaction between type of prenatal supplementation and the offspring genetic background driving GPL-FA levels at school age. Prenatal FO supplementation, and/or with 5-MTHF, seems to stimulate $n-3$ and $n-6$ FA desaturation in the offspring, increasing long-chain PUFA concentrations at school age, but depending on children's FADS1 and FADS2 genotypes. These findings suggest potential early nutrition programming of FA metabolic pathways, but interacting with children's FADS polymorphisms.

Key words: PUFA: Fish-oil: Folic acid: Early programming: Fatty acid desaturase polymorphisms (FADS1, FADS2 and FADS3)

There is consistent evidence that $n-6$ and $n-3$ long-chain (LC) PUFA are important to ensure optimal growth and development, particularly during fetal and early postnatal life $\mathrm{e}^{(1,2)}$.

Desaturation/elongation from essentials linoleic (LA, 18:2n-6) and $\alpha$-linolenic (ALA, C18:3n-3) fatty acids (FA) facilitate the synthesis of important LC-PUFA, including arachidonic acid (AA,
$20: 4 n-6)$, EPA (20:5n-3), docosapentaenoic acid (DPA, $22: 5 n-3)$, and DHA $(22: 6 n-3)^{(3-5)}$. However, the enzymatic machinery involved in this process, including $\Delta^{5}$ and $\Delta^{6}$-desaturases (encoded by fatty acid desaturase (FADS) 1 and FADS2 genes, respectively) and elongases (encoded by elongation of very long chain fatty acids (ELOVL) gene), participates in both pathways,

Disclaimer: This paper was published as part of a supplement to British Journal of Nutrition, publication of which was supported partially by UNILEVER, NUTRIMENTHE EU Project and an unrestricted educational grant from the University of Granada. The papers included in this supplement were invited by the Guest Editor and have undergone the standard journal formal review process. They may be cited.

Abbreviations: 5-MTHF, 5-methyltetrahydrofolate; AA, arachidonic acid; ALA, $\alpha$-linolenic acid; DPA, docosapentaenoic acid; FA, fatty acid; FADS, fatty acid desaturase; FO, fish oil; GPL, glycerophospholipids; LA, linoleic acid; LC, long chain; NUHEAL, Nutraceuticals for a Healthier Life. 
triggering a competition between $n-3$ and $n-6$ series ${ }^{(6)}$. Consequently, inter-individual differences in capacity to form LC-PUFA may depend on the genotype of FADS1 and FADS2 $2^{(7-9)}$, which build a gene cluster on chromosome 11 together with a third desaturase gene, FADS3 $3^{(10,11)}$.

To date, studies have focused on the effects of FA supplementation during pregnancy on body composition, visual and cognitive development ${ }^{(12)}$, as well as effects on immune outcomes and cardiovascular function in early infancy ${ }^{(13,14)}$. Moreover, other authors suggest that fish-oil (FO) or $n-3$ FA supplementation during pregnancy show a potential role on essential FA status in infants ${ }^{(14,15)}$. However, the long-term impact of maternal PUFA status on functionality of the metabolic pathways involved in PUFA desaturation/elongation in children still remains unknown. Interestingly, several studies have demonstrated the association between variants in the human genes FADS1, FADS2 and FADS3 and blood levels of PUFA in European or Caucasian populations ${ }^{(2,3,15-18)}$. It seems that genetic variation in the FADS gene cluster could modify desaturase function supporting that minor alleles increase the concentrations of LA and ALA, and decrease the concentrations of AA, EPA, DPA and DHA in serum, plasma and erythrocytes. Some authors support that $28.5 \%$ of the variability in both PUFA and LC-PUFA levels in human tissues is determined by FADS gene variants ${ }^{(19)}$. However, diets and genetic population background have also a potential role on associations of PUFA levels with different FADS SNP ${ }^{(20,21)}$. Glaser et al. ${ }^{(15)}$ found that FADS1 and FADS2 gene variants modulate tracking of serum PUFA levels in glycerophospholipids (GPL), which are the main lipid constituents of cell membranes with key functions in cell structure, intracellular signalling, anchoring to membranes and stabilisation of protein structure.

With these considerations in mind, we analysed the cheek cell GPL-FA composition in children from the Nutraceuticals for a Healthier Life (NUHEAL) study ${ }^{(22,23)}$ at school age with the following aims:

(1) To analyse if maternal supplementation with DHA and/ or 5-methyltetrahydrofolate (5-MTHF) during pregnancy exerted long-term effects on the LC-PUFA concentrations in their offspring cheek cell GPL at 8, 9 and 9.5 years.

(2) To evaluate whether FADS1, FADS2 and FADS3 genotype polymorphisms may modify PUFA concentrations in schoolchildren's cheek cells.

(3) To explore the possible interaction between the described intervention in the mothers during pregnancy and the offspring FADS polymorphisms on children's LCPUFA concentrations in cheek cell GPL.

\section{Methods \\ Participants}

This study is a part of the European randomised multicentre trial NUHEAL, registered at www.clinicaltrials.gov, reference no. NCT01180933. A total of 270 women took part in the study until giving birth; the detailed study design, subject recruitment and population characteristics have been described elsewhere ${ }^{(22,23)}$. Briefly, NUHEAL is a multicentre randomised study in healthy pregnant women from Munich, Pécs and Granada, designed to assess the effects of receiving daily supplements of FO $(500 \mathrm{mg}$ of DHA $+150 \mathrm{mg}$ EPA), 5-MTHF $(400 \mu \mathrm{g})$, placebo or both $(\mathrm{FO}+5-\mathrm{MTHF})$, from gestation week 20 until delivery, on pregnancy outcomes and on offspring long-term growth and development. Study participants were approached again and asked to participate in a new assessment of their children; 147 complied with the request and cheek cell samples were obtained in all of them at 8, 9 and 9.5 years of age. In all, forty-two (28.6\%) were revisited at the Ludwig-Maximilians-University of Munich, Germany, and 105 (71.4\%) at the University of Granada, Spain. This follow-up examination in the children was performed under the NUTRIMENTHE EU Project framework. Dropout rates were similar between intervention groups, mainly for relocation ( $n$ 3), loss of contact ( $n$ 75) and unwillingness to continue ( $n$ 38). In all, four children were born prematurely before the 35th week of pregnancy, one child was born with a congenital left-side anophthalmus, one child developed craniosynostosis, and another was reported to have left-side deafness. There were no differences in maternal mean dietary intake of DHA between intervention groups at the 20th and 30th weeks of pregnancy. Certain baseline characteristics, including maternal parity, BMI, smoking habits, obstetric risk factors, complications at parturition or perinatal morbidity in any intervention group, did not differ between the studied and not-tested populations. Children whose fathers had a high educational level in the placebo and FO+5MTHF groups showed high drop-out rates $(77.9 \%$ of children with fathers with general qualification for university entrance in the placebo group and $72 \cdot 8 \%$ in the FO +5 -MTHF group withdrew at 8 year follow-up, whereas only $41 \%$ of the fathers of followed children in the placebo group and $32.6 \%$ in the $\mathrm{FO}+5-$ MTHF group had this qualification $(P=0.004)$ ).

The study protocols were approved by the Biomedical Ethical Committees from both participating centres. Written informed consent was obtained from all participants.

\section{Cheek cell sampling procedure}

The sampling procedure consisted of mouth cleaning with $200 \mathrm{ml}$ tap water three times before sampling, followed by rubbing of each inner cheek side with a Bio Brush (reference 73506013; Labolan). Subsequently, the mouth was rinsed with $10 \mathrm{ml}$ pure water, and the rinsing solution containing cells washed off the inner cheeks was collected in a tube. The brush used for scraping was inserted in this tube for a few seconds and, after removing the brush, this tube was closed and centrifuged at $1400 \mathrm{~g}(2710 \mathrm{rpm})$ for $10 \mathrm{~min}$ at $4^{\circ} \mathrm{C}$. The supernatant was discarded. The cell pellet was stored at $-80^{\circ} \mathrm{C}$ until further analysis ${ }^{(24)}$.

\section{Fatty acid measurement from cheek cell glycerophospholipids}

Cheek cell GPL-FA were analysed as described by Klingler et al. ${ }^{\text {(24) }}$ and results are presented as weight percentages of all quantified FA. The sample preparation procedure selectively transferred GPL 
bound FA into their methyl esters, which were quantified by GC. Quality control samples indicated on analytical precision depended on the percentage contribution of the corresponding FA to total GPL-FA and ranged from $2 \cdot 29 \%$ (LA) to $12.53 \%$ (ALA) (CV). After quality control and plausibility checking, cheek cell GPL-FA percentages were compiled in a MS Excel Table.

FA used for the analyses as control were myristic acid (C14:0), palmitic acid (C16:0), margaric acid (C17:0), stearic acid (C18:0), arachidic acid (C20:0), behenic acid (C22:0), lignoceric acid (C24:0), palmitoleic acid (C16:1n-7), vaccenic acid (C18:1n-7), oleic acid (C18:1n-9), eicosaenoic acid (C20:1n-9), nervonic acid (C24:1n-9), LA (C18:2n-6), $\gamma$-linolenic acid (18:3n-6), dihomo- $\gamma$-linolenic acid (C20:3n-6), AA (C20:4n-6), DPA (C22:5n-6), ALA (C18:3n-3), EPA (C20:5n-3), DPA $(\mathrm{C} 22: 5 n-3)$ and DHA (C22:6n-3).

\section{SNP selection and genotyping}

A total of seventeen SNP from the FADS1 (rs174548, rs174556 and rs174561), FADS2 (rs174570, rs174574, rs174575, rs174576, rs174578, rs174579, rs174602, rs498793, rs968567, rs2727271 and rs3834458) and FADS3 (rs174448, rs174449 and rs174455) gene cluster from the children's umbilical cord were genotyped. Of these, eleven SNP were selected according to its localisation in FADS gene cluster on chromosome 11, while six additional SNP (rs174546, rs174561, rs3834458, rs174548, rs174574 and rs174578) were included due to its relation with FA levels previously reported $^{(3,9)}$. The genotyping efficiency was $10 \cdot 7$-fold, although the two further SNP rs174561 and rs3834458 could not be included in the HapMap database. Genotyping of SNP was carried out with the iPLEX method (Sequenom) by means of matrix-assisted laser desorption ionisation-time of the flight MS method (Mass Array; Sequenom), according to the manufacturer's instructions. Standard genotyping quality control included $10 \%$ duplicate and negative samples. The genotyping discordance rate was below $0.3 \%$.

\section{Statistical analyses}

The power calculation was calculated by standard approach, setting $\alpha$ value as 0.05 and $\beta$ value as $0 \cdot 2$. Different studied groups (FO, 5-MTHF, FO+5-MTHF and placebo) were compared with each other for essential FA, including LA, AA, ALA, EPA, $n-5$ DPA and DHA. A statistical power of $80 \%$ for selected FA levels in children's cheek cell GPL at school-age was obtained. Sample size required for our genetic study was also calculated using R package 'powerEQTL version 0.1.3', which considers different ranges of sample sizes, $\alpha$ value of 0.05 and seventeen selected SNP. A statistical power of $90 \%$ was obtained for analysed SNP, except for rs174570 and rs2727271 whose was of $80 \%$ (see online Supplementary Methods for more details).

Histograms of FA levels were studied and assessed for normality using Shapiro-Wilk tests with the help of SPSS statistical program, version 20.0 (SPSS Inc). Some LC-PUFA were extremely right skewed and thus were transformed using the natural logarithm (ln) and square root. If no normal distribution was achieved, non-parametric tests were used. Descriptive results were expressed as means and standard deviations. Categorical outcomes were assessed with $\chi^{2}$ tests and continuous variables with Kruskal-Wallis/one-way ANOVA tests to explore the potential association between FADS polymorphisms and type of prenatal supplementation.

Hardy-Weinberg (H-W) equilibrium for the genotypes was calculated using R statistical software (3.2.2 version, 'genetics' package). Deviations from $\mathrm{H}-\mathrm{W}$ equilibrium were studied using Fisher's exact test. Further, to test the linkage disequilibrium, Lewontin's $D^{\prime}$ and pairwise squared correlations $r^{2}$ were performed.

After selecting the most representative SNP of our sample, the data were transformed into longitudinal form to perform a linear mixed model with the aim to study the relationships between FADS and FA concentrations in cheek cells. The outcomes were the FA concentrations, the fixed part was each one of the selected genotypes (homozygous major allele carriers were coded as 0 , heterozygous as 1 , and homozygous minor allele as 2 ), and the random effect was the time variable ( 8,9 and 9.5 years).

Furthermore, several linear mixed models have been built to assess each FA considering as co-founders the eight selected $\mathrm{SNP}$, group of maternal prenatal supplementation, maternal age at delivery, mother's BMI at weeks 20 and 30 of pregnancy, mother smoking at weeks 20 and 30 of pregnancy, placental weight and sex. To select those co-founder variables, separate analyses were first performed for each of the eight candidate SNP. Then, linear mixed models were adjusted for all other analysed SNP and those covariates which changed between study groups or by SNP. Those co-founders which showed a $P<0.05$ in the preliminary analyses were subsequently included in our final adjusted linear mixed models.

\section{Results}

The baseline characteristics of the study participants are shown in online Supplementary Table S1. There were no significant differences between study groups with respect to sex of the children, maternal age, BMI, smoking at weeks 20 and 30 of pregnancy and placenta weight between the four groups of prenatal supplementation.

\section{Prenatal supplementation and children fatty acids in cheek cell glycerophospholipids}

Depending on mother's supplementation during pregnancy, percentages of those GPL-FA in cheek cells measured in the NUHEAL children resulted statistically significant were presented in Table 1. Our analysis showed a statistical difference in the GLP AA concentrations in cheek cells from 8 to 9.5 year $(P=0 \cdot 002)$. As we can see in Fig. 1, levels of GPL AA significantly decrease from 8 to 9.5 year in those children whose mothers were supplemented with $\mathrm{FO}$ or $\mathrm{FO}+5$-MTHF. In order to understand our results, we have also explored AA:DHA, AA:EPA and AA:[DHA + EPA] ratios, but not significant differences were observed. Moreover, there was also a decrease in AA:DHA ratio from 8 to 9.5 years which did not resulted significant $(P=0.985)$.

At 8 years old, children born to mothers who received the $\mathrm{FO}+5$-MTHF supplementation during pregnancy had higher concentrations of behenic $(P=0.037)$ and $n-6$ DPA acids 
Table 1. Effects of prenatal supplementation on behenic, arachidonic, $n-6$ docosapentaenoic and oleic acid levels in children's cheek cell glycerophospholipids at 8,9 and 9.5 years old $\dagger$

(Mean values and standard deviations)

\begin{tabular}{|c|c|c|c|c|c|c|c|c|c|c|c|c|c|c|}
\hline \multirow[b]{2}{*}{ Fatty acids (\%) } & \multirow{2}{*}{$\begin{array}{l}\text { Supplementation } \\
\text { group }\end{array}$} & \multicolumn{4}{|c|}{8 years } & \multicolumn{4}{|c|}{9 years } & \multicolumn{4}{|c|}{9.5 years } & \multirow[b]{2}{*}{$P 2$} \\
\hline & & $n$ & Mean & SD & $P 1$ & $n$ & Mean & SD & $P 1$ & $n$ & Mean & SD & $P 1$ & \\
\hline \multirow{4}{*}{$\begin{array}{l}\text { Behenic acid } \\
\quad(\mathrm{C} 22: 0)\end{array}$} & 5-MTHF & 27 & 0.24 & 0.12 & \multirow[t]{4}{*}{$0.037^{*}$} & 26 & 0.30 & 0.14 & \multirow[t]{4}{*}{0.751} & 25 & 0.31 & 0.11 & \multirow[t]{4}{*}{0.625} & \multirow[t]{4}{*}{0.325} \\
\hline & FO & 38 & 0.22 & $0 \cdot 10$ & & 33 & 0.34 & $0 \cdot 16$ & & 33 & 0.34 & 0.13 & & \\
\hline & $\mathrm{FO}+5-\mathrm{MTHF}$ & 32 & 0.29 & 0.20 & & 26 & 0.34 & 0.17 & & 29 & 0.33 & 0.15 & & \\
\hline & Placebo & 38 & 0.20 & 0.11 & & 35 & 0.33 & $0 \cdot 17$ & & 35 & 0.30 & 0.09 & & \\
\hline \multirow{4}{*}{$\begin{array}{l}\text { Oleic acid } \\
\qquad(C 18: 1 n-9)\end{array}$} & 5-MTHF & 27 & $24 \cdot 82$ & 4.62 & \multirow[t]{4}{*}{0.829} & 26 & $23 \cdot 81$ & 4.78 & \multirow[t]{4}{*}{$0.035^{\star}$} & 25 & 24.68 & 6.56 & \multirow[t]{4}{*}{0.571} & \multirow[t]{4}{*}{0.325} \\
\hline & FO & 38 & $24 \cdot 80$ & $4 \cdot 22$ & & 33 & $27 \cdot 21$ & $5 \cdot 52$ & & 33 & 24.69 & $5 \cdot 87$ & & \\
\hline & $\mathrm{FO}+5-\mathrm{MTHF}$ & 32 & 24.76 & 5.08 & & 26 & $24 \cdot 22$ & 4.60 & & 29 & $22 \cdot 86$ & 5.02 & & \\
\hline & Placebo & 38 & 23.85 & $6 \cdot 61$ & & 35 & $25 \cdot 11$ & 4.35 & & 35 & 23.76 & 5.65 & & \\
\hline \multirow{4}{*}{$\begin{array}{l}\text { Arachidonic acid } \\
\text { (C20:4n-6) }\end{array}$} & 5-MTHF & 27 & 2.97 & 0.93 & \multirow[t]{4}{*}{0.255} & 26 & $2 \cdot 73$ & 1.08 & \multirow[t]{4}{*}{0.951} & 25 & 2.58 & 0.87 & \multirow[t]{4}{*}{0.962} & \multirow[t]{4}{*}{$0.002^{*}$} \\
\hline & FO & 38 & 3.05 & 0.81 & & 33 & $2 \cdot 66$ & 0.73 & & 33 & $2 \cdot 45$ & 0.80 & & \\
\hline & $\mathrm{FO}+5-\mathrm{MTHF}$ & 32 & $2 \cdot 76$ & 0.94 & & 26 & $2 \cdot 69$ & 0.81 & & 29 & 2.49 & 0.98 & & \\
\hline & Placebo & 38 & $2 \cdot 66$ & 1.04 & & 35 & $2 \cdot 61$ & $0 \cdot 71$ & & 35 & 2.50 & 0.87 & & \\
\hline \multirow{4}{*}{$\begin{array}{l}\text { Docosapentaenoic } \\
\quad \text { acid } \\
\text { (C22:5n-6) }\end{array}$} & 5-MTHF & 27 & 0.08 & 0.04 & \multirow[t]{4}{*}{$0.007^{\star}$} & 25 & 0.16 & 0.09 & \multirow[t]{4}{*}{0.643} & 24 & 0.19 & 0.11 & \multirow[t]{4}{*}{0.939} & \multirow[t]{4}{*}{0.652} \\
\hline & FO & 38 & $0 \cdot 10$ & 0.08 & & 33 & 0.24 & 0.21 & & 33 & 0.20 & 0.21 & & \\
\hline & $\mathrm{FO}+5-\mathrm{MTHF}$ & 32 & 0.20 & 0.16 & & 26 & 0.21 & 0.17 & & 29 & 0.21 & 0.10 & & \\
\hline & Placebo & 38 & 0.07 & 0.03 & & 35 & 0.20 & 0.18 & & 35 & 0.18 & 0.08 & & \\
\hline
\end{tabular}

$n$, number of cases; $P 1$, level of significance from one-way ANOVA analysis for type of prenatal supplementation in each time point; $P 2$, level of significance from one-way ANOVA analysis between 8,9 and 9.5 years old; 5-MTHF, 5-methyltetrahydrofolate; FO, fish oil.

* $P$-values $<0.05$.

$\dagger$ Percentage of total fatty acids from cheek cell glycerophospholipids.

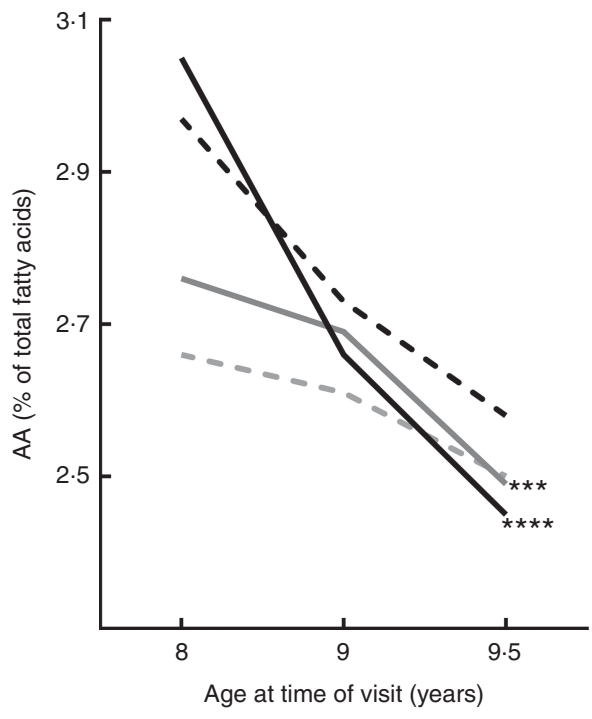

Fig. 1. Evolution of arachidonic acid (AA) concentrations in children's cheek cell glycerophospholipids from 8 to 9.5 years of age, depending on their mothers' supplementation during pregnancy. Significant decrease in AA concentrations were observed in those children born to mothers who received fish oil (FO) with or without 5-methyltetrahydrofolate (5-MTHF). ${ }^{\star \star \star} P<0.001,{ }^{\star \star \star \star} P<0.0001$ after Bonferroni post hoc correction. ——, FO group; ------, 5-MTHF group; - , FO + 5-MTHF group; - - ---, placebo group.

( $P=0.007)$ in cheek cell GPL than those whose mothers were supplemented with FO, 5-MTHF or Placebo. At 9 years old, oleic acid concentrations in GPL measured in cheek cells were higher in those children born to mothers who received FO during pregnancy respect to those born to mothers from 5 -MTHF or FO +5-MTHF groups $(P=0.035)$. Concentrations of other analysed FA, including LA, ALA, EPA, $n$-3 DPA and DHA, did not show significant differences between the four study groups of children at any individual time point (online Supplementary Table S2).

\section{Effects of fatty acid desaturase polymorphisms on fatty acid composition in cheek cells}

Genotype and allele frequencies of seventeen polymorphisms in FADS1, FADS2 and FADS3 genes from NUHEAL children are presented in Table 2 . The analysed genotypes were in $\mathrm{H}-\mathrm{W}$ equilibrium, except for rs174574, rs174576 and rs174578 which were excluded from the following analyses.

Based on the linkage disequilibrium data from Lewontin's $D^{\prime}$ and pairwise-squared correlations $r^{2}$ (Fig. 2 and online Supplementary Table S3) eight SNP out of the initial seventeen SNP (rs174556, rs174570, rs174575, rs174579, rs174602, rs498793, rs2727271 and rs174448) were selected for further analyses. For these selected SNP, Lewontin's $D^{\prime}$ ranged between 0.95 and 1.0 and the pairwise-squared correlations $r^{2}$ ranged between 0.85 and $1 \cdot 0$.

We analysed the effects of FADS gene cluster polymorphisms on GPL-FA concentrations in children. Table 3 represents the regression analysis between the main seven GPL-FA in children cheek cells and the eight FADS gene cluster polymorphisms selected. The analysed FADS1 and FADS 2 polymorphisms showed different associations with LA, ALA, AA, EPA, $n-3$ DPA and DHA concentrations. The majority of the associations were established between FADS1 and FADS2 SNP with LA and ALA. FADS3 rs174448 did not have any effects on the analysed FA concentrations.

The frequencies of major and minor alleles according to genotypes are presented in online Supplementary Table S4. Among all groups of prenatal supplementation, the major allele carriers present highest frequencies for FADS2 and FADS3. No statistical differences were found in the frequencies of major or 
Table 2. Characteristics of the seventeen analysed variants in fatty acid desaturase (FADS) $1 / 2 / 3$ genes of children in the studied population (Numbers and percentages)

\begin{tabular}{|c|c|c|c|c|c|c|c|c|c|}
\hline \multirow[b]{3}{*}{ SNP } & \multirow{3}{*}{$\begin{array}{l}\text { Possible functional } \\
\text { region }\end{array}$} & \multirow{3}{*}{$\begin{array}{c}\begin{array}{c}\text { Alleles (major/minor) } \\
\text { and frequency }\end{array} \\
0 / 1\end{array}$} & \multicolumn{6}{|c|}{ Subjects with genotype } & \multirow[b]{3}{*}{$P$} \\
\hline & & & \multicolumn{2}{|c|}{0} & \multicolumn{2}{|c|}{1} & \multicolumn{2}{|c|}{2} & \\
\hline & & & $n$ & $\%$ & $n$ & $\%$ & $n$ & $\%$ & \\
\hline rs174548 & FADS1 & $\mathrm{G} / \mathrm{C}(0.73 / 0.27)$ & 47 & 0.51 & 41 & 0.45 & 4 & 0.04 & 0.284 \\
\hline rs174556 & FADS1 & $\mathrm{G} / \mathrm{A}(0.74 / 0.26)$ & 49 & 0.53 & 39 & 0.42 & 5 & 0.05 & 0.595 \\
\hline rs174561 & FADS1 & $\mathrm{A} / \mathrm{G}(0 \cdot 75 / 0 \cdot 25)$ & 49 & 0.54 & 37 & 0.41 & \multirow{2}{*}{\multicolumn{2}{|c|}{ ( }} & 0.572 \\
\hline rs174570 & FADS2 & $\mathrm{C} / \mathrm{T}(0.90 / 0.10)$ & 75 & 0.81 & 18 & 0.19 & & & 0.595 \\
\hline rs174574 & FADS2 & $\mathrm{C} / \mathrm{A}(0.68 / 0.32)$ & 38 & 0.41 & 50 & 0.54 & 2 & 0.05 & $0.034^{*}$ \\
\hline rs174575 & FADS2 & $\mathrm{C} / \mathrm{G}(0.73 / 0.27)$ & 47 & 0.51 & 42 & 0.45 & 4 & 0.04 & 0.195 \\
\hline rs174576 & FADS2 & $\mathrm{C} / \mathrm{A}(0.68 / 0.32)$ & 38 & 0.41 & 50 & 0.54 & 5 & 0.05 & $0.034^{*}$ \\
\hline rs174578 & FADS2 & T/A $(0.67 / 0.33)$ & 36 & 0.39 & 51 & 0.55 & 5 & 0.05 & $0.020^{*}$ \\
\hline rs174579 & FADS2 & $\mathrm{C} / \mathrm{T}(0.81 / 0.19)$ & 59 & 0.63 & 33 & 0.35 & 1 & 0.01 & 0.179 \\
\hline rs174602 & FADS2 & $\mathrm{T} / \mathrm{C}(0.77 / 0.23)$ & 53 & 0.57 & 38 & 0.41 & 2 & 0.02 & 0.143 \\
\hline rs498793 & FADS2 & $\mathrm{C} / \mathrm{T}(0.55 / 0.45)$ & 30 & 0.32 & 42 & 0.45 & 21 & 0.23 & 0.406 \\
\hline rs968567 & FADS2 & $\mathrm{C} / \mathrm{T}(0.83 / 0 \cdot 17)$ & 61 & 0.66 & 32 & 0.34 & \multicolumn{2}{|c|}{-} & 0.064 \\
\hline rs2727271 & FADS2 & $\mathrm{A} / \mathrm{T}(0.89 / 0 \cdot 11)$ & 73 & 0.78 & 20 & 0.22 & \multicolumn{2}{|c|}{ - } & 0.591 \\
\hline rs3834458 & FADS2 & $\mathrm{T} / \mathrm{Z}(0 \cdot 70 / 0 \cdot 30)$ & 42 & 0.45 & 46 & 0.49 & 5 & 0.05 & 0.138 \\
\hline rs174448 & FADS3 & $\mathrm{T} / \mathrm{C}(0.69 / 0.31)$ & 42 & 0.45 & 45 & 0.48 & 6 & 0.06 & 0.229 \\
\hline rs174449 & FADS3 & $\mathrm{T} / \mathrm{C}(0.69 / 0.31)$ & 41 & 0.44 & 46 & 0.49 & 6 & 0.06 & 0.225 \\
\hline rs174455 & FADS3 & $\mathrm{T} / \mathrm{C}(0.68 / 0.32)$ & 41 & 0.45 & 43 & 0.47 & 8 & 0.09 & 0.633 \\
\hline
\end{tabular}

0 , homozygous major; 1 , heterozygous; 2 , homozygous minor; $P$, level of significance according to Fisher's exact test. ${ }^{*} P$-values $<0.05$.

(a)

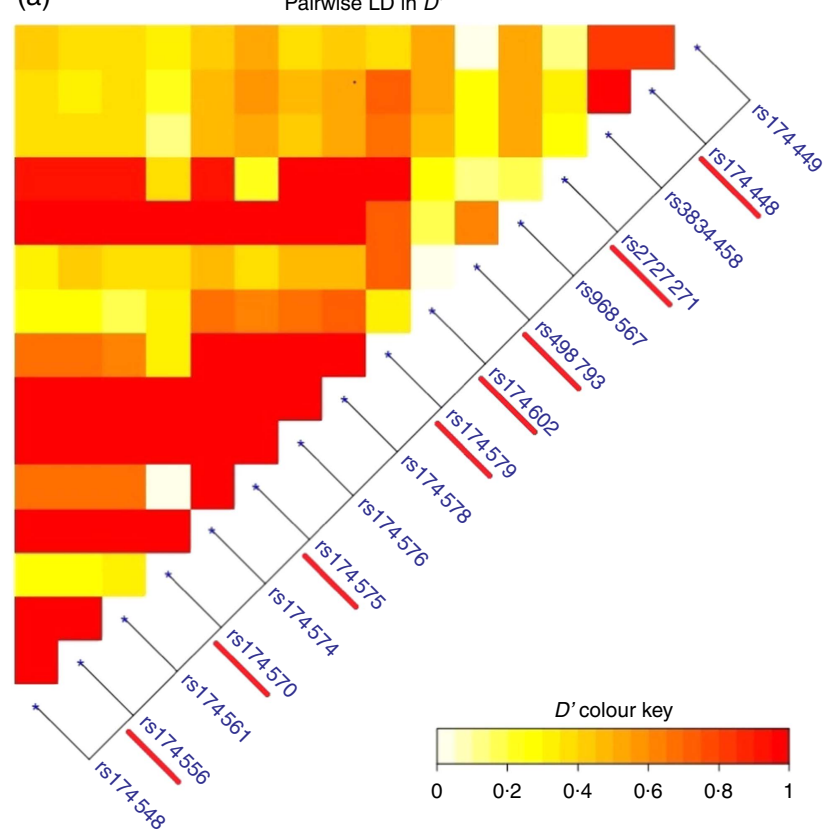

(b)

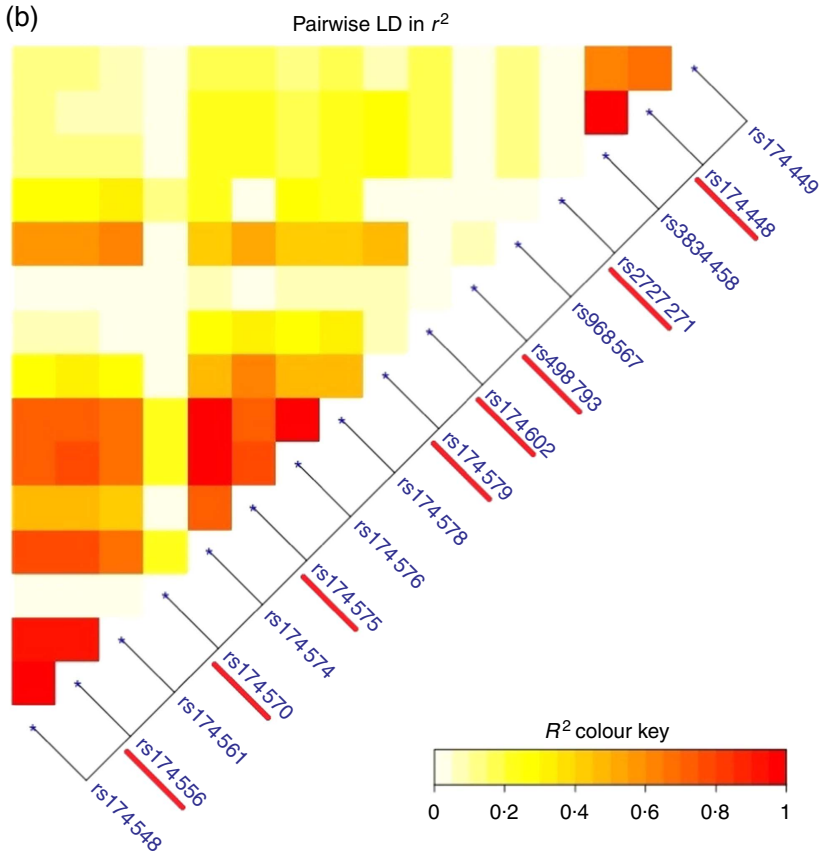

Fig. 2. (a) Pairwise linkage disequilibrium (LD) measured in $D^{\prime}$ for the complete seventeen SNP obtained from Nutraceuticals for a Healthier Life (NUHEAL) children; (b) pairwise LD measured in $r^{2}$ for the complete seventeen SNP obtained from NUHEAL children.

minor homozygous and heterozygous for FADS1, FADS2 or FADS3 between children depending on their mothers groups of supplementation.

Next, we analysed the effects of SNP on FA concentration depending on the maternal supplementation groups (Table 4).

As shown in Table 4, LA children whose mothers were supplemented with FO, and carrying the minor homozygous of both FADS1 rs174556 and FADS2 rs174575 showed high LA concentrations respect to heterozygosis or major homozygous genotypes. High LA levels were also found in those children born to mothers who received placebo and carrying the heterozygous genotype for FADS1 rs174556, FADS2 rs174575 and rs174579. AA concentrations were increased in children with FADS2 rs174570 and rs174579 major homozygous genotypes in case of maternal FO or placebo supplementation, respectively. No other effects of the different polymorphisms or supplementation type were observed. 
Table 3. Associations between PUFA concentrations in children's cheek cell glycerophosphopholipids and selected SNP of fatty acid desaturase (FADS) gene cluster polymorphism

( $\beta$-Coefficients and $95 \%$ confidence intervals)

\begin{tabular}{|c|c|c|c|c|c|c|c|c|c|}
\hline & & \multirow{2}{*}{$\frac{F A D S 1}{r s 174556}$} & \multicolumn{6}{|c|}{ FADS2 } & \multirow{2}{*}{$\frac{F A D S 3}{\text { rs174448 }}$} \\
\hline & & & rs 174570 & rs174575 & rs174579 & rs174602 & rs498793 & rs2727271 & \\
\hline \multirow[t]{4}{*}{ Linoleic acid (C18:2n-6) } & Int & 15.79 & $16 \cdot 30$ & $15 \cdot 80$ & $16 \cdot 02$ & 16.07 & $16 \cdot 91$ & $16 \cdot 22$ & $16 \cdot 35$ \\
\hline & $\beta$ & 1.19 & 0.28 & 1.19 & 1.14 & 0.73 & -0.51 & 0.85 & 0.09 \\
\hline & $95 \% \mathrm{Cl}$ & $0.57,1.81$ & $-0.64,1.24$ & $0.55,1.84$ & $0.38,1.90$ & $0.03,1.43$ & $-1.04,-0.01$ & $-0.05,1.75$ & $-0.54,0.74$ \\
\hline & $P$ & $<0.001^{*}$ & 0.558 & $<0.001^{\star}$ & $0.003^{*}$ & $0.039^{\star}$ & 0.057 & 0.065 & 0.765 \\
\hline \multirow[t]{4}{*}{ Arachidonic acid (C20:4n-6) } & Int & $2 \cdot 71$ & $2 \cdot 74$ & 2.77 & $2 \cdot 27$ & $2 \cdot 74$ & $2 \cdot 82$ & 2.63 & $2 \cdot 74$ \\
\hline & $\beta$ & -0.06 & -0.31 & -0.18 & -0.31 & -0.13 & -0.15 & 0.20 & -0.10 \\
\hline & $95 \% \mathrm{Cl}$ & $-0.25,0.12$ & $-0.60,-0.03$ & $-0.38,0.005$ & $-0.53,-0.08$ & $-0.34,0.07$ & $-0.30,0.006$ & $-0.06,0.47$ & $-0.30,0.08$ \\
\hline & $P$ & 0.489 & $0.028^{\star}$ & 0.057 & $0.007^{\star}$ & 0.193 & 0.068 & 0.143 & 0.272 \\
\hline \multirow{4}{*}{$\begin{array}{l}\text { Docosapentaenoic acid } \\
\text { (C22:5n-6) }\end{array}$} & Int & 0.22 & 0.41 & 0.28 & 0.17 & 0.32 & 0.29 & 0.21 & 0.39 \\
\hline & $\beta$ & 0.11 & 0.15 & -0.14 & -0.21 & -0.13 & -0.21 & 0.54 & -0.21 \\
\hline & $95 \% \mathrm{Cl}$ & $0.22,0.42$ & $-0.02,0.22$ & $-0.41,0.51$ & $0.24,0.54$ & $-0.41,0.17$ & $-0.39,0.09$ & $0.12,0.71$ & $-0.41,0.15$ \\
\hline & $P$ & 0.514 & 0.065 & 0.412 & 0.221 & 0.544 & 0.24 & 0.094 & 0.234 \\
\hline \multirow[t]{4}{*}{$a$-Linolenic acid (C18:3n-3) } & Int & 0.29 & 0.32 & 0.28 & 0.30 & 0.31 & 0.33 & 0.32 & 0.34 \\
\hline & $\beta$ & 0.07 & 0.02 & 0.08 & 0.07 & 0.04 & -0.01 & 0.02 & -0.02 \\
\hline & $95 \% \mathrm{Cl}$ & $0.01,0.12$ & $-0.05,0.10$ & $0.02,0.13$ & $0.01,0.14$ & $-0.01,0.10$ & $-0.05,0.03$ & $-0.05,0.10$ & $-0.08,0.02$ \\
\hline & $P$ & $0.009^{*}$ & 0.527 & $0.005^{\star}$ & $0.020^{*}$ & 0.177 & 0.659 & 0.494 & 0.314 \\
\hline \multirow{4}{*}{ EPA (C20:5n-3) } & Int & $0 \cdot 17$ & 0.33 & 0.27 & 0.26 & 0.27 & 0.34 & 0.15 & 0.31 \\
\hline & $\beta$ & 0.10 & 0.16 & -0.08 & -0.10 & -0.10 & -0.12 & 0.33 & -0.14 \\
\hline & $95 \% \mathrm{Cl}$ & $0.12,0.32$ & $-0.04,0.67$ & $-0.32,0.44$ & $0.37,0.17$ & $-0.35,0.14$ & $-0.31,0.06$ & $0.09,0.65$ & $-0.37,0.08$ \\
\hline & $P$ & 0.374 & 0.053 & 0.455 & 0.475 & 0.416 & 0.201 & $0.044^{\star}$ & 0.224 \\
\hline \multirow{4}{*}{$\begin{array}{l}\text { Docosapentaenoic acid } \\
\text { (C22:5n-3) }\end{array}$} & Int & 0.23 & 0.21 & 0.32 & 0.31 & 0.33 & 0.40 & 0.21 & -0.13 \\
\hline & $\beta$ & 0.08 & 0.30 & -0.09 & -0.10 & -0.11 & -0.12 & 0.30 & 0.36 \\
\hline & $95 \% \mathrm{Cl}$ & $-0.12,0.30$ & $-0.01,0.62$ & $-0.31,0.12$ & $-0.36,0.15$ & $-0.35,0.12$ & $-0.30,0.05$ & $0.001,0.60$ & $-0.35,0.08$ \\
\hline & $P$ & 0.411 & 0.064 & 0.412 & 0.429 & 0.336 & $0 \cdot 161$ & $0.049^{*}$ & 0.222 \\
\hline \multirow[t]{4}{*}{ DHA (C22:6n-3) } & Int & 0.65 & 0.62 & 0.76 & 0.73 & 0.77 & 0.78 & 0.61 & 0.79 \\
\hline & $\beta$ & 0.09 & 0.35 & -0.13 & $-0 \cdot 11$ & $-0 \cdot 17$ & -0.09 & 0.34 & -0.15 \\
\hline & $95 \% \mathrm{Cl}$ & $-0.18,0.31$ & $-0.01,0.73$ & $-0.39,0.11$ & $-0.41,0.18$ & $-0.45,0.09$ & $-0.30,0.11$ & $-0.01,0.70$ & $-0.40,0.09$ \\
\hline & $P$ & 0.595 & 0.058 & 0.293 & 0.437 & 0.199 & 0.361 & 0.058 & 0.224 \\
\hline
\end{tabular}

Int, intercept (constant from regression model) (mean fatty acid concentration in homozygous carriers of the major allele); $\beta$, regression coefficient (linear regression of the fatty acid (outcome) on the respective single SNP); $P$, level of significance obtained from linear regression.

* $P$-values $<0.05$.

Prenatal 5-MTHF or FO + 5-MTHF supplementation and specific FADS polymorphisms significantly affected $n-6$ DPA levels. In fact, those children whose mothers received prenatal 5-MTHF supplementation, and carrying the major homozygous genotypes for FADS3 rs174448, showed high n-6 DPA levels. Regarding prenatal FO+5-MTHF supplementation, we observed higher $n$-6 DPA levels in children with minor homozygous of FADS2 rs174575 and rs174602. Interestingly, higher $n-6$ DPA concentrations were found in those children born to mothers who received placebo and carrying the major homozygotic alleles for FADS2 rs174602 and rs2727271 polymorphisms.

Those children, whose mothers received FO supplementation during pregnancy, and having the minor homozygotic alleles for FADS1 rs174556 and FADS2 rs174575 had a higher level of ALA in cheek cell GPL, respect to those carrying the other polymorphisms. Furthermore, children carrying the heterozygous genotypes for rs174579, whose mothers were supplemented with 5-MTHF, showed an increase of ALA concentrations in cheek cell GPL. Higher ALA levels were also found in those children heterozygous for FADS2 rs174575 and rs174579 variants born to mothers who received placebo during pregnancy.

Those children heterozygous for the FADS2 rs174570 polymorphism, and whose mothers received placebo during pregnancy, had a lower concentration of EPA in cheek cell GPL, compared with those children carrying major homozygous; prenatal FO+5-MTHF supplementation was found to be associated with high levels of EPA in those heterozygous children carrying FADS2 rs2727271 or minor homozygosis in FADS2 rs174602 polymorphisms. Children born to mothers who were supplemented with FO had higher DPA concentrations in cheek cell GPL when they carried the major homozygous rs174579 and heterozygous rs2727271. Moreover, prenatal supplementation with FO+5-MTHF determined higher concentrations of DPA when children were heterozygous for FADS2 rs498793. On the other hand, high DHA levels are caused by major homozygous FADS2 rs174602 and heterozygous FADS1 rs174556 and FADS2 rs174575 in those children who mothers received prenatal FO supplementation. No definitive pattern was found in both DPA and DHA concentrations in those children born to mothers who received placebo; in this case, the presence of the different FADS polymorphisms had no effect on aforementioned FA levels.

Finally, we analysed the effects of SNP and different confounders on the FA concentrations in GPL from cheek cells using a lineal mixed model (Table 5). LA concentrations were influenced by rs174556, rs174602, rs498793 and rs174448 genotypes, child's sex, maternal age and BMI $20 \mathrm{~kg} / \mathrm{m}^{2}(P<0 \cdot 0001)$. ALA concentrations in cheek cell GPL were associated with rs174570, rs174448 and sex. AA was influenced by all the SNP studied, except rs174602, rs498793 and rs174448. Moreover, we did not observe any association between $n-6$ DPA levels and selected confounders. 
Table 4. Relationship between fatty acid glycerophospholipid concentrations in children's cheek cells and fatty acid desaturase (FADS) gene cluster polymorphisms by type of prenatal supplementation $\dagger$ (Number of samples, mean values and standard deviations)

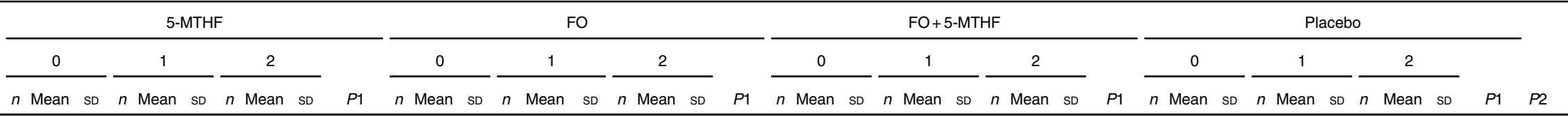

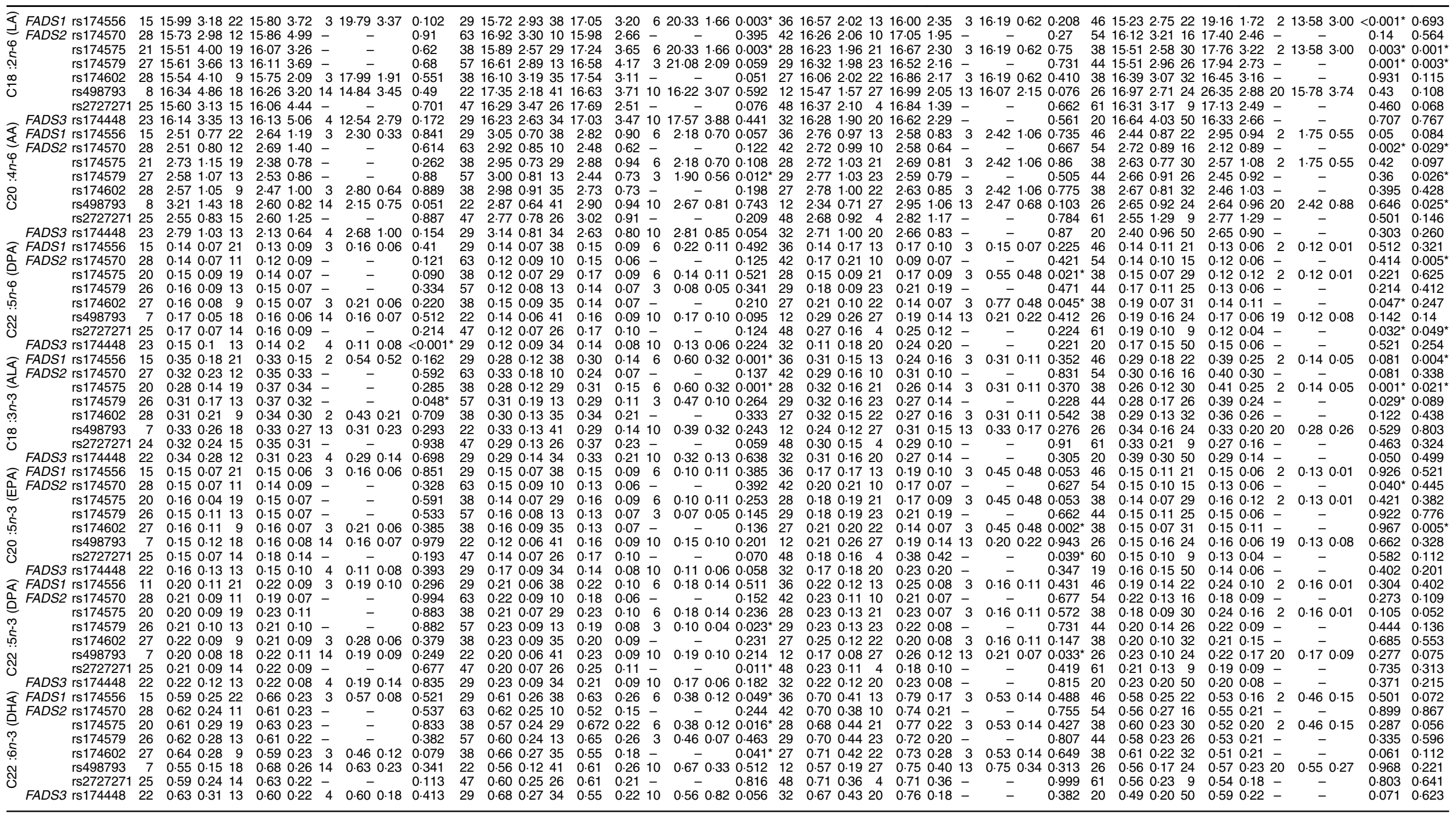

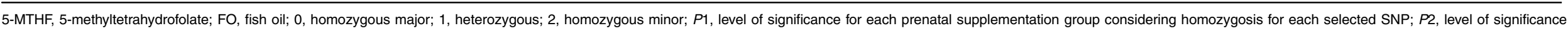
between prenatal supplementation groups; LA, linoleic acid; AA, arachidonic acid; DPA, docosapentaenoic acid; ALA, $a$-linolenic acid.
* $P$-value $<0.05$.

† Random effect $=$ time points ( 8,9 and 9.5 years of age). 
Table 5. Associations between PUFA in glycerophospholipids from children's cheek cell samples and selected SNP of fatty acid desaturase gene cluster polymorphism adjusted by different co-founders ( $\beta$-Coefficients and $95 \%$ confidence intervals)

\begin{tabular}{|c|c|c|c|c|c|c|c|c|c|c|c|c|c|c|c|c|c|c|c|c|}
\hline & & CONS & rs 174556 & rs 174570 & rs174575 & rs174579 & rs174602 & rs 498793 & rs2727271 & rs174448 & Sex & MA & BMI20 & BMI30 & $\mathrm{S} 20$ & S30 & PWD & GS & $P$ value & $\begin{array}{c}P \text { value } \\
\text { adjusted }\end{array}$ \\
\hline \multirow[t]{4}{*}{ LA } & $\beta$ & $19 \cdot 13$ & 1.53 & 0.36 & -0.50 & 0.33 & 1.02 & -0.63 & -0.82 & -1.06 & $-1 \cdot 20$ & 0.15 & -0.49 & 0.18 & 1.40 & -0.27 & 0.00 & 0.09 & $<0.0001^{*}$ & $<0.0001^{*}$ \\
\hline & $95 \% \mathrm{Cl}$ low & 13.98 & 0.06 & -0.90 & -1.94 & -1.73 & 0.05 & $-1 \cdot 19$ & -3.06 & -1.76 & $-1 \cdot 19$ & 0.06 & -0.94 & -0.23 & 0.31 & -1.73 & -0.01 & -0.24 & & \\
\hline & $95 \% \mathrm{Cl}$ high & 24.27 & 3.08 & 1.64 & 0.93 & 2.41 & $2 \cdot 01$ & -0.08 & 1.42 & -0.36 & -0.42 & 0.24 & -0.03 & 0.60 & $3 \cdot 13$ & $1 \cdot 17$ & 0.04 & 0.43 & & \\
\hline & $P$ & $<0.01^{*}$ & $0.04^{*}$ & 0.57 & 0.49 & 0.74 & $0.03^{*}$ & $0.02^{\star}$ & 0.47 & $<0.01^{*}$ & $<0.01^{*}$ & $<0.01^{*}$ & $0.03^{*}$ & 0.39 & 0.10 & 0.71 & 0.88 & 0.59 & & \\
\hline \multirow[t]{4}{*}{ AA } & $\beta$ & $4 \cdot 82$ & -0.44 & 0.80 & -0.55 & 0.62 & 0.06 & -0.09 & 0.79 & 0.11 & -0.45 & 0.01 & -0.18 & 0.14 & -0.13 & -0.41 & -0.01 & -0.01 & $<0.0001^{*}$ & $<0.0001^{*}$ \\
\hline & $95 \% \mathrm{Cl}$ low & 3.24 & -0.90 & $-1 \cdot 19$ & -0.99 & -0.01 & -0.23 & -0.26 & 0.10 & -0.10 & -0.69 & -0.01 & -0.32 & 0.01 & -0.66 & -0.86 & -0.10 & $-0 \cdot 10$ & & \\
\hline & $95 \% \mathrm{Cl}$ high & $6 \cdot 24$ & 0.1 & -0.40 & -0.11 & 1.25 & 0.36 & 0.07 & 1.48 & 0.32 & -0.22 & 0.04 & -0.04 & 0.27 & 0.39 & 0.03 & 0.10 & 0.1 & & \\
\hline & $P$ & $<0.01^{*}$ & $0.05^{*}$ & $<0.01^{*}$ & $0.01^{*}$ & $0.05^{*}$ & 0.67 & 0.28 & $0.02^{*}$ & 0.31 & $<0.01^{*}$ & 0.33 & $<0.01^{*}$ & $0.02^{*}$ & 0.60 & 0.07 & 0.26 & 0.59 & & \\
\hline \multirow[t]{4}{*}{$n-6$ DPA } & $\beta$ & 0.35 & -0.02 & -0.014 & -0.074 & 0.044 & 0.01 & 0.15 & 0.11 & -0.05 & -0.03 & 0.001 & -0.04 & 0.04 & 0.01 & -0.07 & 0.15 & 0.01 & 0.145 & 0.0667 \\
\hline & $95 \% \mathrm{Cl}$ low & 0.1 & -0.1 & -0.09 & -0.06 & -0.07 & -0.03 & -0.01 & -0.04 & -0.04 & -0.05 & -0.05 & -0.07 & -0.02 & -0.06 & -0.08 & -0.00 & -0.01 & & \\
\hline & $95 \% \mathrm{Cl}$ high & 0.4 & 0.02 & 0.04 & 0.14 & 0.21 & 0.05 & 0.02 & 0.25 & 0.05 & 0.03 & 0.05 & 0.1 & 0.07 & 0.08 & 0.08 & 0.05 & 0.21 & & \\
\hline & $P$ & $0.01^{*}$ & 0.21 & 0.05 & 0.41 & 0.87 & 0.85 & 0.67 & 0.069 & 0.24 & 0.15 & 0.65 & 0.56 & 0.78 & 0.88 & 0.33 & 0.87 & 0.52 & & \\
\hline \multirow[t]{4}{*}{ ALA } & $\beta$ & 0.82 & 0.10 & 0.10 & 0.08 & -0.10 & -0.01 & -0.02 & -0.12 & -0.05 & 0.06 & 0.03 & -0.01 & -0.01 & 0.12 & -0.05 & 0.00 & -0.17 & $<0.0001^{*}$ & $<0.0001^{\star}$ \\
\hline & $95 \% \mathrm{Cl}$ low & 0.42 & -0.01 & 0.01 & -0.02 & -0.26 & -0.08 & -0.06 & -0.29 & -0.11 & -0.12 & -0.04 & -0.05 & -0.05 & -0.01 & -0.16 & -0.01 & -0.05 & & \\
\hline & $95 \% \mathrm{Cl}$ high & 1.20 & 0.01 & 0.20 & 0.19 & 0.05 & 0.06 & 0.02 & 0.05 & -0.04 & -0.05 & 0.1 & 0.02 & 0.03 & 0.25 & 0.05 & -0.01 & -0.1 & & \\
\hline & $P$ & $<0.01^{\star}$ & 0.07 & $0.03^{*}$ & 0.14 & 0.19 & 0.79 & 0.31 & 0.17 & $0.03^{*}$ & $0.03^{*}$ & 0.39 & 0.49 & 0.59 & 0.07 & 0.33 & 0.63 & 0.19 & & \\
\hline \multirow[t]{4}{*}{ EPA } & $\beta$ & 0.25 & -0.03 & -0.04 & -0.04 & 0.06 & 0.00 & 0.05 & 0.09 & -0.01 & -0.02 & 0.00 & -0.01 & 0.01 & 0.04 & -0.03 & 0.00 & 0.00 & 0.2810 & $0.0437^{*}$ \\
\hline & $95 \% \mathrm{Cl}$ low & 0.05 & -0.09 & -0.09 & -0.06 & -0.01 & -0.03 & -0.01 & 0.04 & -0.04 & -0.05 & -0.01 & -0.03 & -0.05 & -0.06 & -0.08 & -0.00 & -0.01 & & \\
\hline & $95 \% \mathrm{Cl}$ high & 0.45 & 0.01 & 0.01 & 0.04 & 0.14 & 0.03 & 0.02 & 0.17 & 0.01 & 0.00 & 0.01 & 0.03 & 0.02 & 0.06 & 0.02 & 0.01 & 0.01 & & \\
\hline & $P$ & $0.01^{*}$ & 0.19 & 0.06 & 0.83 & 0.13 & 0.99 & 0.67 & $0.04^{*}$ & 0.34 & 0.09 & 0.66 & 0.11 & 0.19 & 0.91 & 0.27 & 0.97 & 0.99 & & \\
\hline \multirow[t]{4}{*}{$n-3$ DPA } & $\beta$ & 0.44 & -0.03 & -0.03 & -0.00 & 0.03 & -0.01 & -0.08 & 0.05 & -0.01 & -0.03 & 0.04 & -0.01 & 0.08 & -0.03 & -0.03 & -0.00 & -0.04 & $<0.0001^{*}$ & $<0.0001^{*}$ \\
\hline & $95 \% \mathrm{Cl}$ low & 0.27 & -0.08 & -0.07 & -0.04 & -0.03 & -0.05 & -0.02 & -0.02 & -0.03 & -0.06 & 0.00 & -0.02 & -0.06 & -0.08 & -0.08 & -0.00 & -0.01 & & \\
\hline & $95 \% \mathrm{Cl}$ high & 0.61 & 0.01 & 0.01 & 0.04 & 0.10 & 0.01 & 0.01 & 0.1 & -0.01 & -0.01 & 0.01 & 0.01 & 0.02 & 0.02 & 0.01 & 0.00 & 0.07 & & \\
\hline & $P$ & $<0.01^{*}$ & 0.20 & 0.08 & 0.98 & 0.32 & 0.26 & 0.41 & 0.16 & 0.32 & $<0.01^{*}$ & $0.02^{*}$ & 0.06 & 0.27 & 0.26 & 0.16 & 0.24 & 0.51 & & \\
\hline \multirow[t]{4}{*}{ DHA } & $\beta$ & 0.67 & -0.24 & -0.14 & -0.09 & 0.34 & -0.03 & 0.06 & 035 & 0.02 & -0.04 & 0.04 & -0.02 & 0.01 & -0.07 & $0 . .01$ & 0.00 & -0.02 & 0.0899 & 0.2068 \\
\hline & $95 \% \mathrm{Cl}$ low & 0.15 & -0.39 & -0.26 & -0.24 & 0.13 & -0.13 & 0.05 & 0.12 & -0.04 & -0.11 & -0.05 & -0.11 & -0.06 & -0.02 & -0.24 & -0.00 & -0.06 & & \\
\hline & $95 \% \mathrm{Cl}$ high & 1.18 & -0.09 & -0.01 & 0.04 & 0.55 & 0.06 & 0.11 & 0.57 & 0.09 & 0.03 & 0.1 & 0.03 & 0.01 & 0.06 & 0.09 & 0.00 & 0.08 & & \\
\hline & $P$ & $0.01^{*}$ & $<0.01^{*}$ & $0.03^{\star}$ & 0.17 & $<0.01^{*}$ & 0.51 & $0.03^{*}$ & $<0.01^{*}$ & 0.47 & 0.25 & 0.41 & 0.31 & 0.40 & 0.39 & 0.98 & 0.34 & 0.13 & & \\
\hline
\end{tabular}

CONS, constant value; MA, maternal age; BMI20, BMI at 20 weeks of pregnancy; BMI30, BMI at 30 weeks of pregnancy; S20, smoking at 20 weeks of pregnancy; S30, smoking at 30 weeks of pregnancy; PWD, placental weight at delivery; GS, group of prenatal supplementation; $P$, level of significance of each co-founder variable; $P$-value, unadjusted level of significance considering all analysed co-founders; $P$-value adjusted, level of significance considering selected co-founders; LA, linoleic acid; AA, arachidonic acid; DPA, docosapentaenoic acid; ALA, $a$-linoleic acid.

* $P$-value $<0.05$ 
Regarding $n$ - 3 series LC-PUFA, EPA shows a liaison with rs2727271, meanwhile $n-3$ DPA shows no correlation with any SNP, but with sex and maternal age. Different SNP influenced the DHA concentrations, but the model showed no statistical significance.

\section{Discussion}

This is the first study suggesting the presence of long-term effects of maternal prenatal supplementation with DHA and/or 5-MTHF on PUFA status and metabolism in the offspring at school age; furthermore, our data also seem to indicate an interaction between maternal supplementation during pregnancy and their offspring polymorphisms in the FADS cluster, determining children's PUFA concentrations in cheek cell GPL at school age.

Previous results from NUHEAL study demonstrate clear associations between maternal FA concentrations in plasma phospholipids during pregnancy and those levels found in their offspring (umbilical cord) ${ }^{(1)}$, as well as changes depending on maternal supplementation during pregnancy. However, the present analysis suggests long-term effects of maternal supplementation with $\mathrm{FO}$ or $\mathrm{FO}+5$-MTHF during pregnancy on the FA status in cheek cell GPL in their offspring up to 9.5 years. In fact, supplementation with $\mathrm{FO}+5$-MTHF during pregnancy determined an increase of children's $n-6$ DPA concentrations in cheek cell GPL at 8 years old, suggesting an additive effect of both supplements promoting a major activity in the $n-6$ PUFA series. It is also important to notice that children's AA and DHA concentrations measured in cheek cell GPL from 8 to 9.5 years are within normal ranges, but a significant decrease of AA concentrations was observed in those children whose mothers were supplemented with $\mathrm{FO}$ or $\mathrm{FO}+5$-MTHF. Furthermore, there were no significant differences for AA:DHA, AA:EPA and $\mathrm{AA}:[\mathrm{DHA}+\mathrm{EPA}]$ ratios. Thus, our results suggest that maternal FO prenatal supplementation could determine a beneficial programming effect on the metabolic pathways during fetal life, which would be maintained up to late childhood; specifically, this supplement would program the metabolic $n-6$ FA pathways with a significant reduction of the synthesis of AA without affecting $n-6$ concentrations or aforementioned ratios. This apparent beneficial effect could be related to a lower risk of adverse health consequences in the offspring in later life. As our study is not able to demonstrate if this effect is linked to an excess of maternal DHA and EPA intake during pregnancy, our results should be interpreted with caution due to the sample size and the multiple confounding factors along 9.5 years of life, but still are of interest for future studies. In this regard, a recent experimental study in newborn and recently weaned offspring mice supported that changes in specific hepatic microRNA (miRNA) (miR-122 and $m i R-370$, respectively) are independently determined by an excess of maternal lipids during both gestational and lactation periods, leading in turn to impaired lipid metabolism in the offspring ${ }^{(25)}$. Finally, we suggest that this decline may also be associated with slightly modifications in dietary intake of AA, LA or $n$ - 3 LC-PUFA, which have a role in increasing or decreasing the AA concentrations. In fact, we observed an increase of $n$-3 LC-PUFA intake from 8 to 9 years in both German and Spanish children (data not shown).
Prenatal supplementation with FO alone also determined higher oleic acid concentrations in GPL in the offspring at 9.5 years old, respect to those children born to mothers who received 5-MTHF. Again the interaction between FO and 5-MTHF seems to have long-term effects on the biochemical pathways involved in FA and folate metabolisms.

Our study also tried to evaluate whether FADS1, FADS2 and FADS3 genotype polymorphisms may modify PUFA concentrations in schoolchildren's cheek cells. To date, findings support that $28.5 \%$ of the variability observed in both PUFA and LC-PUFA levels in human tissues are associated with FADS gene variants $^{(1,19)}$; therefore, it is clear that both diet and genetic variation play an important role in determining blood and tissue levels of LA and ALA, as well as their biologically active LCPUFA derivatives. In fact, this is the first study suggesting the associations of genetic variants in the FADS1, FADS2 and, to a lesser extent, FADS3 gene cluster and children's cheek cell GPLFA. Interestingly, our study results seem to indicate a long-term interaction between maternal supplementation during pregnancy and FADS genotypes that extends up to 9.5 years of age; the effect of such potential interaction may determine $n-3$ and n-6 LC-PUFA concentrations in childhood. Maternal supplementation with FO alone or plus 5-MTHF was associated with both $n$ - 3 and $n$-6 LC-PUFA status in their offspring cheek cell GPL; however, supplementation only with FO or 5-MTHF, upon a possible interaction with $F A D S$ polymorphisms mainly causes an increase in the concentration of ALA. This pattern is also seen in infants born to mothers supplemented with placebo, except for $n-3$ DPA and DHA levels. In the Avon Longitudinal Study of Parents and Children (ALSPAC), child's FADS genotypes influenced amounts of cord plasma LC-PUFA and FA ratios; furthermore, the authors suggested a specific effect of the minor allele on FA metabolism ${ }^{(9)}$.

The present data suggest that maternal FO supplementation in association with minor homozygosis for FADS1 rs174556 and FADS2 rs174575 determine a decrease of FADS activity and subsequent increase of $n-3$ and $n-6$ FA precursors. However, other FADS 2 polymorphisms seem to be associated with significant decrease of AA, $n$-3 DPA and DHA in cheek cell GPL. Interestingly, 5-MTHF supplementation during pregnancy did not show any effect on desaturation and elongation of both $n-3$ and $n$ - 6 FA in the offspring, independently of the FADS gene polymorphisms; only, major homozygosis of FADS3 rs174448 was associated with higher $n-6$ DPA and heterozygosis of FADS2 rs174579 with higher ALA concentrations. To date, it is reported that folic acid improves the re-methylation of homocysteine, which leads to the formation of methionine. Some authors ${ }^{(26)}$ have suggested that methionine stimulates phosphatidylethanolamine methylation, thereby altering the ratio of phosphatidylcholine to phosphatidylethanolamine in liver microsomes; as consequence, $\Delta^{5}$ and $\Delta^{6}$-acyl-coenzyme A desaturases increase their enzymatic activity, which in turn results in higher LC-PUFA concentrations. Our results show that the supplement combining $\mathrm{FO}+5$-MTHF during pregnancy was not associated with the same effect than FO alone on cheek cell GPL-FA in the offspring up to 9.5 years; on the contrary, changes in allele frequencies in FADS2 in the FO+5-MTHF group seem to be associated with an increase in $n-6$ DPA, $n-3$ DPA and EPA. With these results we are just speculating that there seems to be an interaction between 
early life supplementation and offspring FADS polymorphisms determining FA status at childhood. Consequently, future studies are needed to clarify this interaction, evaluating also the potential effects of ELOVL gene polymorphisms, together with FADS gene cluster, to disentangle all actors involved in the potential early programming of LC-PUFA metabolic pathways.

It is important to highlight that FA status is largely based on analysis of FA levels in plasma, erythrocytes and adipose tissue. These approaches, however, are invasive and not well accepted in population studies, particularly in those including infants and young children. In the present study, we measured FA in cheek cells as reference of FA status. Despite its limitations in sample quality and quantity, buccal mucosal cheek cell phospholipids should be proposed as a non-invasive biological marker for LC-PUFA status in children ${ }^{(24)}$. In fact, several studies have previously reported that cheek cell FA composition not only reflects dietary FA intake, but also both erythrocyte and plasma levels of essential PUFA ${ }^{(27-30)}$. In this regard, we found that daily intake of AA, EPA and folate increase the levels of AA, EPA and $n-5$ DPA in GPL of children's cheek cells, while DHA intake decreases the concentration of $n-5$ DPA (data not shown). Furthermore, several authors had already demonstrated the association between variants in the FADS1, FADS2, FADS3 genes and blood levels of PUFA ${ }^{(2,3,15-18)}$. On one side, PUFA concentrations were strongly associated with higher or lower prevalence of several diseases, including the metabolic syndrome ${ }^{(31)}$, obesity $^{(32,33)}, \mathrm{CVD}^{(34,35)}$, atopic sensitisations ${ }^{(36,37)}$ or rheumatoid $\operatorname{arthritis}^{(38-40)}$. On the other side, FADS polymorphisms have not only shown potential effects on neuropsychological and developmental outcomes in infants ${ }^{(17,41)}$, but also on FA related disorders, including attention-deficit/hyperactivity disorder, allergic diseases and the metabolic syndrome ${ }^{(18)}$. With these considerations in mind, we suggest that the profile of FA in cheek cell GPL would be an easy method in children to study whether interaction between FA status and the FADS polymorphisms may be related to the development or evolution of some diseases.

Besides, LC-PUFA status can be considered as a biomarker of the effect of supplementation during pregnancy, which proves again that there is a long-term effect of early nutrition in the offspring $^{(1)}$. We demonstrated that prenatal supplementation with FO and 5-MTHF has a long-term effect on cognitive (attention) development in the offspring at 8 years ${ }^{(42)}$, but we should seriously consider that may be a potential impact on neurodevelopment depending on children's own lipid metabolism. Moreover, other authors noticed that specific PUFA may have also general health benefits ${ }^{(16,43)}$, but may depend on the individual FADS genotypes. So, it will be very interesting to perform further studies to evaluate this hypothesis in different fields of children's health.

Finally, we believe it is important to mention that the results of our current study have an added value, because to our knowledge, no study has made such a long-term follow-up after a prenatal supplementation. However, in the current study there are limitations that must be acknowledged. First, higher number of cases per group would have been better, but the sample size achieved resulted high enough to detect relevant FA concentrations differences between groups. Furthermore, we did not see a positive or negative trend in our data. Although sample size for genetic study was about $80-90 \%$, we are all aware that larger sample would have led us to find more compelling results. Having in mind these considerations, we think that the current study is still interesting as a pilot to opening new perspectives focused on the role of both genotype and early nutrition on the long-term development of FA metabolism.

Secondly, the effects of FO and 5-MTHF supplementation during pregnancy on the obtained results at school age should be interpreted with caution, due to the short window of data collection. Current international recommendations for pregnant women indicate that they must achieve at least $200-300 \mathrm{mg}$ of $\mathrm{DHA} / \mathrm{d}^{(44,45)}$. Considering that NUHEAL study included two groups of pregnant women who received daily $500 \mathrm{mg}$ DHA $+150 \mathrm{mg}$ EPA (FO) from week 20 until delivery, and the suggested joint action between FO and 5-MTHF supplements, the potential excess of LC-PUFA intake in some women would have long-term unknown consequences. Taken into account these results, we propose that those women who follow a diet rich in both DHA and folic acid should receive an individualised supplement based on their genetic characteristics and real needs, avoiding the excess of these nutrients. In this regard, it is possible that both DHA and folic acid show specific critical windows during pregnancy which do not necessarily coincide. Thus, future studies are needed to further investigate the potential benefits of both DHA and folic acid supplementation at different times of gestation, avoiding overdoses and interaction between both nutrients to favouring fetal development without potential long-term adverse effects.

On the other side, children were programmed to be examined twice within NUTRIMENTHE framework, when they were aged 7.5 and 9 years; due to the long-time required to fulfil the protocol agreed, each assessment was divided into two visits resulting in four visits at 7.5-8 and 9-9.5 years old. So, finally we took cheek cell samples four times during 2 years, coinciding with the four visits performed; these repeated samples' collection improved the accuracy and reliability of this new methodology. However, cheek cell samples were obtained at 7.5 years with a different type of brush, and therefore were discarded.

In summary, herewith we report that $\mathrm{FO}$ or $\mathrm{FO}+5$-MTHF supplementation during pregnancy seems to have long-term effects on the GPL-FA profile in the cheek cells of the offspring. Most of the FADS polymorphisms studied are associated with PUFA measured in the NUHEAL children's cheek cells. Together, data obtained from this study suggest that long-term FA status during childhood is not only related to early nutrition programming of FA metabolic pathways, but also FADS genetic variations in children. Further research is guaranteed to demonstrate, in a more plausible evolutionary design, the longterm effects of early life nutritional interventions depending on individual FADS genetic variations.

\section{Acknowledgements}

The authors are grateful to Pilar Brandi and Gudrun Haile for their collaboration in contacting the families, administering the questionnaires and collecting the samples. The authors thank all people involved in the huge number of tasks of this study. Moreover, the authors thank the participants for their generous collaboration 
for such a long time and the heads of the institutions (German, Hungarian and Spanish) to ideate this wonderful project, NUHEAL.

This work was supported by the Commission of the European Community's Seventh Framework Program (FP7/2008-2013), grant agreement no. 212652 (NUTRIMENTHE Project), Marie Curie post-doctoral fellowship (FP7, no. 329812, NutriOmics); within the Sixtth Framework Program, contract no. 007036 (EARNEST Project); and supported in part by the Commission of the European Community within the Fifth Framework Program, contract no. QLK1-CT-1999-00888 (NUHEAL EU project). This publication is the work of the authors and does not necessarily reflect the views of the Commission of the European Community. The work of B. K. is supported by the European Research Council Advanced Grant ERC-2012-AdG - no. 322605 METAGROWTH. Funders had no role in the design, analysis or writing of this article.

All authors helped in the interpretation of results and contributed to manuscript preparation. C. C. coordinated and designed the study, was the coordinator of the study at University of Granada and wrote the manuscript; C. M.-Z. and H. D. performed the cheek cell analysis; C. M.-Z. and J. A. G.-S. wrote the manuscript. E. R. and P. R. have been responsible for the FADS polymorphisms analysis. C. C., C. M.-Z., S. A. and H. A. worked on the data analysis and interpretation; B. K. was the coordinator of the NUHEAL study in Germany and supervised the manuscript.

The results reported in this article are likely to be included in the Doctoral Thesis of H.A. at the University of Granada, PhD Program in Epidemiology and Public Health.

The authors declare that there are no conflicts of interest.

\section{Supplementary materials}

For supplementary material/s referred to in this article, please visit https://doi.org/10.1017/S0007114518002623

\section{References}

1. Escolano-Margarit MV, Campoy C, Ramirez-Tortosa MC, et al. (2013) Effects of fish oil supplementation on the fatty acid profile in erythrocyte membrane and plasma phospholipids of pregnant women and their offspring: a randomised controlled trial. Br J Nutr 109, 1647-1656.

2. Harslof LB, Larsen LH, Ritz C, et al. (2013) FADS genotype and diet are important determinants of DHA status: a cross-sectional study in Danish infants. Am J Clin Nutr 97, 1403-1410.

3. Koletzko B, Lattka E, Zeilinger S, et al. (2011) Genetic variants of the fatty acid desaturase gene cluster predict amounts of red blood cell docosahexaenoic and other polyunsaturated fatty acids in pregnant women: findings from the Avon Longitudinal Study of Parents and Children. Am J Clin Nutr 93, 211-219.

4. Molto-Puigmarti C, Plat J, Mensink RP, et al. (2010) FADS1 FADS2 gene variants modify the association between fish intake and the docosahexaenoic acid proportions in human milk. Am J Clin Nutr 91, 1368-1376.

5. Park WJ, Kothapalli KS, Reardon HT, et al. (2012) A novel FADS1 isoform potentiates FADS2-mediated production of eicosanoid precursor fatty acids. J Lipid Res 53, 1502-1512.

6. de la Garza PA, Montes GR, Chisaguano Tonato AM, et al. (2017) Association of maternal weight with FADS and ELOVL genetic variants and fatty acid levels - the PREOBE follow-up. PLOS ONE 12, e0179135.

7. Yeates AJ, Love TM, Engstrom K, et al. (2015) Genetic variation in FADS genes is associated with maternal long-chain PUFA status but not with cognitive development of infants in a high fish-eating observational study. Prostaglandins Leukot Essent Fatty Acids 102-103, 13-20.

8. Barman M, Nilsson S, Torinsson NA, et al. (2015) Single nucleotide polymorphisms in the FADS gene cluster but not the ELOVL2 gene are associated with serum polyunsaturated fatty acid composition and development of allergy (in a Swedish Birth Cohort). Nutrients 7, 10100-10115.

9. Lattka E, Koletzko B, Zeilinger S, et al. (2013) Umbilical cord PUFA are determined by maternal and child fatty acid desaturase (FADS) genetic variants in the Avon Longitudinal Study of Parents and Children (ALSPAC). Br J Nutr 109, 1196-1210.

10. Lattka E, Illig T, Koletzko B, et al. (2010) Genetic variants of the FADS1 FADS2 gene cluster as related to essential fatty acid metabolism. Curr Opin Lipidol 21, 64-69.

11. Reardon HT, Hsieh AT, Park WJ, et al. (2013) Dietary long-chain polyunsaturated fatty acids upregulate expression of FADS3 transcripts. Prostaglandins Leukot Essent Fatty Acids 88, 15-19.

12. Campoy C, Escolano-Margarit MV, Anjos T, et al. (2012) Omega 3 fatty acids on child growth, visual acuity and neurodevelopment. Br J Nutr 107, Suppl. 2, S85-S106.

13. Drewery ML, Gaitan AV, Spedale SB, et al. (2017) Maternal n-6 and $n-3$ fatty acid status during pregnancy is related to infant heart rate and heart rate variability: an exploratory study. Prostaglandins Leukot Essent Fatty Acids 126, 117-125.

14. Rodriguez-Santana Y, Ochoa JJ, Lara-Villoslada F, et al. (2017) Cytokine distribution in mothers and breastfed children after omega-3 LCPUFAs supplementation during the last trimester of pregnancy and the lactation period: a randomized, controlled trial. Prostaglandins Leukot Essent Fatty Acids 126, 32-38.

15. Glaser C, Heinrich J \& Koletzko B (2010) Role of FADS1 and FADS2 polymorphisms in polyunsaturated fatty acid metabolism. Metabolism 59, 993-999.

16. Lattka E, Klopp N, Demmelmair H, et al. (2012) Genetic variations in polyunsaturated fatty acid metabolism-implications for child health? Ann Nutr Metab 60, Suppl. 3, S8-S17.

17. Lauritzen L, Sorensen LB, Harslof LB, et al. (2017) Mendelian randomization shows sex-specific associations between longchain PUFA-related genotypes and cognitive performance in Danish schoolchildren. Am J Clin Nutr 106, 88-95.

18. Meldrum SJ, Li Y, Zhang G, et al. (2017) Can polymorphisms in the fatty acid desaturase (FADS) gene cluster alter the effects of fish oil supplementation on plasma and erythrocyte fatty acid profiles? An exploratory study. Eur J Nutr 19, 2583-2594.

19. Schaeffer L, Gohlke H, Muller M, et al. (2006) Common genetic variants of the FADS1 FADS2 gene cluster and their reconstructed haplotypes are associated with the fatty acid composition in phospholipids. Hum Mol Genet 15, 1745-1756.

20. Gonzalez-Casanova I, Rzehak P, Stein A, et al. (2016) Maternal single nucleotide polymorphisms in the fatty acid desaturase 1 and 2 coding regions modify the impact of prenatal supplementation with DHA on birth weight. Am J Clin Nutr 103, 1171-1178.

21. Tanjung C, Rzehak P, Mansyur M, et al. (2017) Study protocol to investigate the environmental and genetic aetiology of atopic dermatitis: the Indonesian Prospective Study of Atopic Dermatitis in Infants (ISADI). BMJ Open 7, e012475.

22. Campoy C, Escolano-Margarit MV, Ramos R, et al. (2011) Effects of prenatal fish-oil and 5-methyltetrahydrofolate supplementation on cognitive development of children at 6.5 year of age. Am J Clin Nutr 94, Suppl. 6, S1880-S1888.

23. Krauss-Etschmann S, Shadid R, Campoy C, et al. (2007) Effects of fish-oil and folate supplementation of pregnant women on 
maternal and fetal plasma concentrations of docosahexaenoic acid and eicosapentaenoic acid: a European randomized multicenter trial. Am J Clin Nutr 85, 1392-1400.

24. Klingler M, Demmelmair H, Koletzko B, et al. (2011) Fatty acid status determination by cheek cell sampling combined with methanol-based ultrasound extraction of glycerophospholipids. Lipids 46, 981-990.

25. de Paula Simino LA, de Fante T, Figueiredo M, et al. (2017) Lipid overload during gestation and lactation can independently alter lipid homeostasis in offspring and promote metabolic impairment after new challenge to high-fat diet. Nutr Metab (Lond) 14, 16.

26. Sugiyama K, Kumazawa A, Zhou H, et al. (1998) Dietary methionine level affects linoleic acid metabolism through phosphatidylethanolamine $N$-methylation in rats. Lipids 33, 235-242.

27. Hoffman DR, Birch EE, Birch DG, et al. (1999) Fatty acid profile of buccal cheek cell phospholipids as an index for dietary intake of docosahexaenoic acid in preterm infants. Lipids 34, 337-342.

28. Connor SL, Zhu N, Anderson GJ, et al. (2000) Cheek cell phospholipids in human infants: a marker of docosahexaenoic and arachidonic acids in the diet, plasma, and red blood cells. Am J Clin Nutr 71, 21-27.

29. Laitinen K, Sallinen J, Linderborg K, et al. (2006) Serum, cheek cell and breast milk fatty acid compositions in infants with atopic and non-atopic eczema. Clin Exp Allergy 36, 166-173.

30. Kirby A, Woodward A, Jackson S, et al. (2010) A double-blind, placebo-controlled study investigating the effects of omega-3 supplementation in children aged 8-10 years from a mainstream school population. Res Dev Disabil 31, 718-730.

31. Guo XF, Li X, Shi M, et al. (2017) n-3 Polyunsaturated fatty acids and metabolic syndrome risk: a meta-analysis. Nutrients 9, E703.

32. Simopoulos AP (2016) An increase in the omega-6/omega-3 fatty acid ratio increases the risk for obesity. Nutrients $\mathbf{8}, 128$.

33. Vaittinen M, Mannisto V, Kakela P, et al. (2017) Interorgan cross talk between fatty acid metabolism, tissue inflammation, and FADS2 genotype in humans with obesity. Obesity $\mathbf{2 5}$, $545-552$.

34. Endo J \& Arita M (2016) Cardioprotective mechanism of omega-3 polyunsaturated fatty acids. J Cardiol 67, 22-27.
35. Takahashi M, Ando J, Shimada K, et al. (2017) The ratio of serum $n-3$ to $n-6$ polyunsaturated fatty acids is associated with diabetes mellitus in patients with prior myocardial infarction: a multicenter cross-sectional study. BMC Cardiovasc Disord 17, 41.

36. Miles EA \& Calder PC (2017) Can early omega-3 fatty acid exposure reduce risk of childhood allergic disease? Nutrients 21, E784.

37. Rucci E, den Dekker HT, de Jongste JC, et al. (2016) Maternal fatty acid levels during pregnancy, childhood lung function and atopic diseases. The Generation R Study. Clin Exp Allergy 46, 461-471.

38. Brouwers H, von HJ, Toes R, et al. (2015) Lipid mediators of inflammation in rheumatoid arthritis and osteoarthritis. Best Pract Res Clin Rheumatol 29, 741-755.

39. Rodriguez-Carrio J, Alperi-Lopez M, Lopez P, et al. (2016) Nonesterified fatty acids profiling in rheumatoid arthritis: associations with clinical features and Th1 response. PLOS ONE 11, e0159573.

40. Zhou J, Chen J, Hu C, et al. (2016) Exploration of the serum metabolite signature in patients with rheumatoid arthritis using gas chromatography-mass spectrometry. J Pharm Biomed Anal 127, 60-67.

41. Andersen KR, Harslof LB, Schnurr TM, et al. (2017) A study of associations between early DHA status and fatty acid desaturase (FADS) SNP and developmental outcomes in children of obese mothers. Br J Nutr 117, 278-286.

42. Catena A, Muñoz-Machicao JA, Torres-Espinola FJ, et al. (2016) Folate and long-chain polyunsaturated fatty acid supplementation during pregnancy has long-term effects on the attention system of 8.5 year-old offspring: a randomized controlled trial. Am J Clin Nutr 103, 115-127.

43. Chisaguano AM, Montes R, Perez-Berezo T, et al. (2013) Gene expression of desaturase (FADS1 and FADS2) and elongase (ELOVL5) enzymes in peripheral blood: association with polyunsaturated fatty acid levels and atopic eczema in 4-yearold children. PLOS ONE 8, e78245.

44. Koletzko B, Cetin I \& Brenna JT (2007) Dietary fat intakes for pregnant and lactating women. Br J Nutr 98, 873-877.

45. Koletzko B, Lien E, Agostoni C, et al. (2008) The roles of longchain polyunsaturated fatty acids in pregnancy, lactation and infancy: review of current knowledge and consensus recommendations. J Perinat Med 36, 5-14. 